\title{
Structure of Nascent Microbial Cellulose II. Effects of Methyl and Methoxy Groups of Direct Blue 14 and 15 on Nascent Microbial Cellulose
}

\author{
Md. Ibrahim H. MondAL ${ }^{\dagger}$ and Akira $\mathrm{KAI}^{\dagger \dagger}$ \\ Department of Industrial Chemistry, Tokyo Metropolitan University, \\ Minami-Ohsawa, Hachioji, Tokyo 192-03, Japan
}

(Received March 31, 1997)

\begin{abstract}
The influence of Direct Blue 14 and Direct Blue 15 having the same skeletal backbone structure except difference of methyl and methoxy groups, numbers and positions of other substituent groups on the structure of nascent microbial cellulose was examined. The products from Acetobacter-culture in the presence of Direct Blue 14 or Direct Blue 15, and celluloses regenerated from these products are almost the same in respect of structure and nature characterized by X-ray, solid state ${ }^{13} \mathrm{C}$ NMR and deuteration-IR measurements. Each of the products is a crystalline dye-cellulose complex and regenerated cellulose is cellulose II. From these results, it is clear that the effect of methyl groups in Direct Blue 14 on the nascent microbial cellulose is almost the same as that of methoxy groups in Direct Blue 15.

KEY WORDS Acetobacter xylinum / Microbial Cellulose / Dye Cellulose Complex / Cellulose Sheet /

Direct Blue 14 / Direct Blue 15 / Regenerated Cellulose /
\end{abstract}

Microbial cellulose (MC) is of current interest to scientists. The main reasons are: (a) cellulose is mostly pure without lignin; (b) cellulose is of great mechanical strength; (c) and can be modified directly during synthesis. (d) It is comparatively easy to study the formation mechanism; and (e) volume and rate of product can be controlled. In these interests, our main aim is (c) and (d). In the previous paper, ${ }^{1-3}$ we reported the structure of nascent MC through modification by the use of direct dyes. Direct dyes have different effects on the structure of MC depending on the structures of dyes and substituent groups on the skeletal backbone as well as position. The number of sulfonate groups in a dye affect the conformation of cellulose chains, consequently, cellulose $\mathrm{I} \alpha$ rich to other cellulose allomorphs, e.g., cellulose $\mathrm{I} \beta,{ }^{4} \mathrm{II},{ }^{3,5} \mathrm{IV}_{\mathrm{I}},{ }^{1,2,6}$ and the position of sulfonate groups affect the fine structure of cellulose in the same allomorphs. ${ }^{2,5}$

This paper reports the effects of methyl and methoxy groups in Direct Blue 14 and Direct Blue 15 (DB14 and DB15) having the same biphenylenebis(azo) skeletal structure on the structure of nascent MC. In the previous paper, ${ }^{5}$ we reported the effects of these groups in DB1 and DB53 on the structure of nascent MC. The differences of the former groups with the latter groups are only the positions of sulfonate groups on the same skeletal backbone. The former groups contains sulfonate groups at 3 and 6 in the naphthalene nucleus while the latter groups, at 5 and 7 in the naphthalene nucleus. The position of sulfonate group has some effect on the fine structure, ${ }^{3}$ and thus we make a comparative study between these groups of dyes. The structures of the product as well as the regenerated cellulose were characterized by X-ray diffractometer, solid state ${ }^{13} \mathrm{C}$ NMR and deuteration-IR spectrometers.

\section{EXPERIMENTAL}

\section{Preparation of Samples}

Product Sample. Acetobacter xylinum (IFO 13693) was cultured in a $15 \mathrm{~cm}$ diameter petridish containing 110 $\mathrm{ml}$ Hestrin-Schramm medium ${ }^{7}\left(\mathrm{pH}\right.$ 6.8) at $28^{\circ} \mathrm{C}$ for about 3 days in an incubator. Sixty $\mathrm{ml}$ of cellulose-free cell suspension, prepared from matured petridish in $120 \mathrm{ml}$ phosphate buffer solution, were added to $140 \mathrm{ml}$ of HS medium ( $\mathrm{pH} 7.0$ ) containing a direct dye of $0.1 \mathrm{wt} \%$ and the mixture was incubated at $28^{\circ} \mathrm{C}$ for $24 \mathrm{~h}$ in a static conditions. ${ }^{5}$ The product was collected by centrifuging $(6000 \mathrm{rpm}, 5.15 \mathrm{~g}, 20 \mathrm{~min})$ and washed well with distilled water to remove the dye not related to dyeing. These products were preserved at $3^{\circ} \mathrm{C}$ in the wet. The direct dyes used in this experiments are DB14 $\left(2,2^{\prime}-\left[4,4^{\prime}-\left(3,3^{\prime}-\right.\right.\right.$ dimethyl)biphenylenebis(azo)]bis[8-amino-1-hydroxynaphthalene-3,6-disulfonic acid] tetrasodium salt; Aldrich Chemical Co.) and DB15 (2,2'-\{4,4'-[3,3-bis(methoxy)]biphenylenebis(azo) $\}$ bis [8-amino-1-hydroxynaphthalene-3,6-disulfonic acid]tetrasodium salt); Aldrich Chemical Co.). The chemical structure of these dyes are shown in Fig. 1.

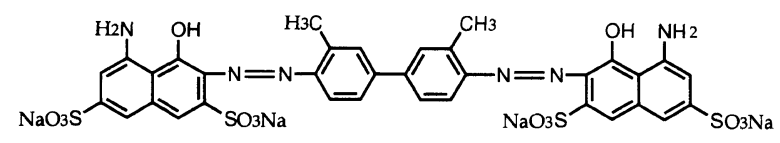

Direct Blue 14

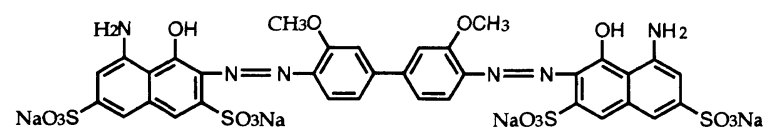

Direct Blue 15

Figure 1. The chemical structures of direct dyes.

\footnotetext{
† Present address: Department of Applied Chemistry and Chemical Technology, Rajshahi University, Rajshahi, Bangladesh.

${ }^{\dagger \dagger}$ To whom correspondence should be addressed.
} 
Regenerated Cellulose Sample. The dye extraction of the above samples was performed by boiling in $70 \mathrm{vol} \%$ aqueous ethanol solutions for $18 \mathrm{~h}$ and fresh aqueous ethanol solution was exchanged every $3 \mathrm{~h}$. This dye extracted sample was again boiled in $1.0 \mathrm{wt} \%$ aqueous sodium hydroxide solution for $10 \mathrm{~h}$ under $\mathrm{N}_{2}$ atmosphere. The regenerated cellulose was neutralized with $1.0 \mathrm{vol} \%$ aqueous acetic acid and rinsed with distilled water. The sample was preserved as above. ${ }^{5}$

Control Cellulose Samples. Control cellulose I was prepared by washing $\mathrm{MC}$ with distilled water to remove the medium components and boiled in $1.0 \mathrm{wt} \%$ aqueous sodium hydroxide solution for $10 \mathrm{~h}$ under $\mathrm{N}_{2}$ atmosphere. The final sample (cellulose I or MC) was neutralized and rinsed as above. The cellulose II sample was prepared by mercerization of $\mathrm{MC}$ at $20^{\circ} \mathrm{C}$.

\section{Methods for the Measurements}

$X$-Ray Measurements. To understand the structures of products from Acetobacter-culture in the presence of DB14 or DB15 and of the cellulose regenerated from it, the following measurements were performed.

The X-ray diffraction diagram of an uniplanar oriented membrane of product was measured by the reflection and transmission. The membrane was prepared from the product and was dried on a Teflon plate at room temperature. The diffraction diagram of an isotropic product and regenerated cellulose was obtained by the transmission method. In this method, the samples were compressed as pellets into the pores of a sample holder of $2 \mathrm{~mm}$ diameter and $1 \mathrm{~mm}$ thickness. The X-ray diffractogram was measured by a $\mathrm{MXP}^{18}$ diffractometer (MAC Science) with $\mathrm{Ni}$-filtered $\mathrm{Cu}-K_{\alpha}$ radiation. The measurement conditions were as followed: divergence slit, $1.0 \mathrm{~mm}$; receiving slit, $0.15 \mathrm{~mm}$; scanning speed, $4.0^{\circ} \mathrm{min}^{-1}$; X-ray radiation, $40 \mathrm{kV}, 200 \mathrm{~mA}$.

Solid State ${ }^{13} \mathrm{C}$ NMR Spectroscopy. Solid state ${ }^{13} \mathrm{C}$ NMR experiments were performed at room temperature on a JEOL JNM EX 270 spectrometer operating at $6.35 \mathrm{~T}$, as described elsewhere. ${ }^{2,3}$ The MAS rate was $5.0-5.5 \mathrm{kHz}$, and the chemical shifts relative to tetramethylsilane were determined using the crystalline peak at $17.3 \mathrm{ppm}$ of solid hexamethyl benzene as the external standard. The non-dried samples were packed in a MAS rotor with an O-ring sealed to avoid the loss of water during NMR measurements. The spectrum of the crystalline component of control and regenerated celluloses was measured selectively by taking NMR signals after relaxation of the noncrystalline component. The signal obtained after $\tau=50$ was measured using T1CP pulse sequence $^{8}$ with CP.

Deuteration-IR Spectroscopy. To obtain IR spectra of the sample, a membrane suitable for IR spectra (about $10 \mu \mathrm{m}$ in dry thickness) was prepared from the nondried preserved samples and dried on a Teflon plate. Deuteration was performed by the method described previously. ${ }^{9,10}$ The sample membrane was set inside a glass cell so that the IR beam would be perpendicular to the membrane surface. Before deuteration, the glass cell with the sample was dried under reduced pressure $\left(10^{-3}\right.$ Torr $)$ for $3 \mathrm{~h}$, and then vapour phase deuteration of the sample was performed by $\mathrm{D}_{2} \mathrm{O}(99.8 \%$; Aldrich Chemical Co.) for a given time at room temperature.

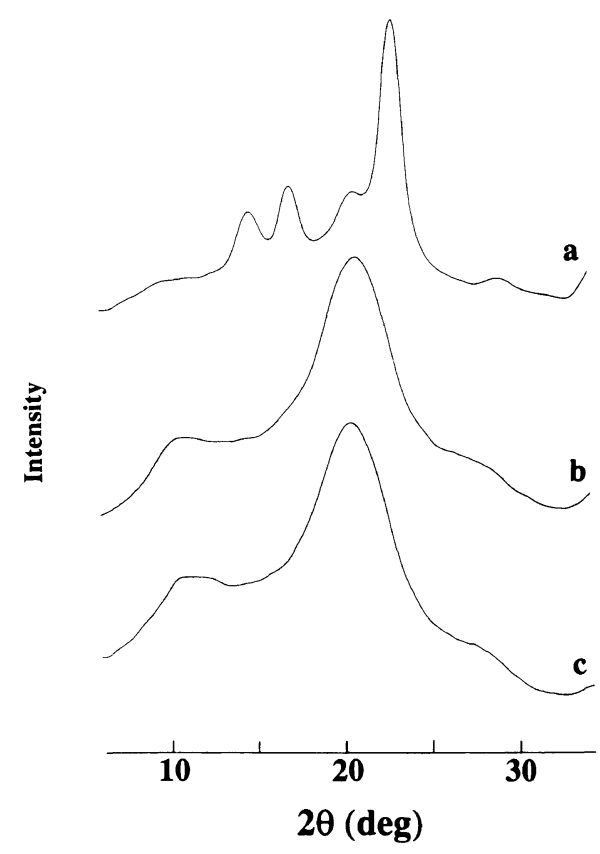

Figure 2. X-Ray diffractograms of microbial cellulose and products from Acetobacter-culture in the presence of a dye of $0.1 \mathrm{wt} \%$. a, MC; b, DB14 product; c, DB15 product.

Table I. X-Ray diffraction angles and $d$-spacing of MC and products obtained from Acetobacter-culture in the presence of DB14 or DB15

\begin{tabular}{|c|c|c|c|c|c|}
\hline \multirow{2}{*}{$\begin{array}{l}\text { Sample } \\
\mathrm{MC}\end{array}$} & \multicolumn{5}{|c|}{$\begin{array}{c}20 / \text { deg } \\
(d \text {-spacing in } \AA)\end{array}$} \\
\hline & - & $\begin{array}{l}14.5(w) \\
(6.12)\end{array}$ & $\begin{array}{l}16.8(w) \\
(5.28)\end{array}$ & $20.4(w)$ & $\begin{array}{l}22.7(\mathrm{~s}) \\
(3.92)\end{array}$ \\
\hline DB14 & $\begin{array}{l}10.1(\mathrm{vw}) \\
(8.76)\end{array}$ & 一 & - & $\begin{array}{l}20.6(\mathrm{~s}) \\
(4.32)\end{array}$ & \\
\hline DB15 & $\begin{array}{l}10.4(w) \\
(8.53)\end{array}$ & - & - & $\begin{array}{l}20.3(\mathrm{~s}) \\
(4.36)\end{array}$ & \\
\hline
\end{tabular}

The deuterated sample was again dried under reduced pressure, and the IR spectrum was measured. FT-IR systems 800 (Nicolet) was used for the IR spectroscopy. The amounts of $\mathrm{OH}$ and OD groups were calculated as described previously. ${ }^{2}$

\section{RESULTS AND DISCUSSION}

\section{Structure of Product Samples}

Figure 2 shows $\mathrm{X}$-ray diffraction diagrams of $\mathrm{MC}$ and the product obtained from Acetobacter-culture in the presence of DB 14 or DB 15 (DB14 or DB15 product). $2 \theta$ of the diffraction angles of MC, DB14, and DB15 products are listed in Table I.

From the diffraction diagrams in Figure 2 and diffraction angles in Table I, the diffraction diagrams of products differ apperently from that of MC. Although each product has two major diffraction peaks at near 10 and $20.5^{\circ}$ and the diffraction pattern is broader compared to $\mathrm{MC}$, the diagram indicates that the product is in a crystalline state. This suggests that the product is a crystalline complex of a dye and cellulose, and the normal hydrogen bonding of MC in the product is hindered by the dye molecules. 
Md. I. H. Mondal and A. KaI

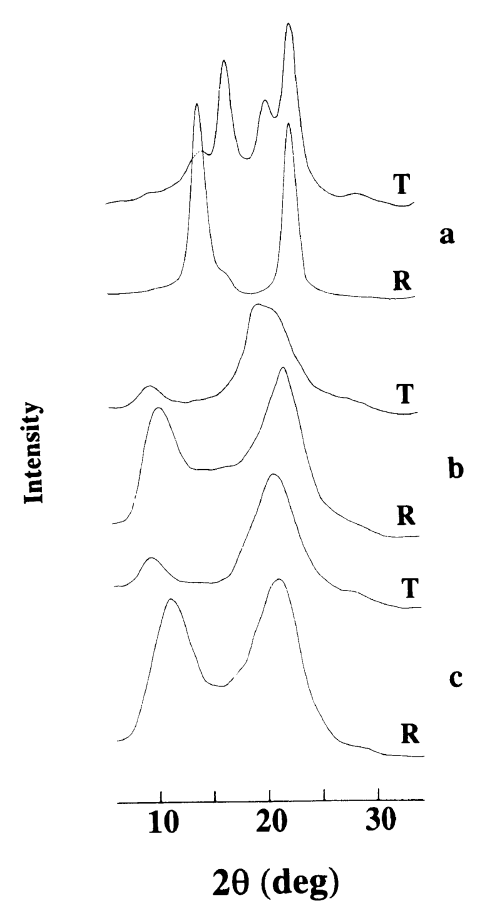

Figure 3. X-Ray diffractograms of oriented membranes of microbial cellulose and products measured by transmission $(\mathrm{T})$ and reflection (R). a, MC; b, DB14 product; c, DB15 product.

The diffraction diagrams of DB14 and DB15 product membranes measured by transmission and reflection methods are shown in the Figure 3. The diffraction diagram of DB14 product membrane measured by transmission or reflection is similar to that of DB15 product membrane. In both product membranes, when measured by reflection, the diffraction of lower angle plane becomes stronger than that measured by transmission. The higher angle peak does not change in intensity in reflection or transmission. The diffraction peak of lower angle plane of product membrane is not strong as that of MC when measured by the reflection method, but this plane seems parallel to the surface. In the case of the oriented membrane of MC, the (1110) plane of $\mathrm{MC}$ is preferentially parallel to the membrane surface, and the (110) plane is perpendicular to the surface. ${ }^{11}$ In the case of complexes of Fluorescent Brightener ${ }^{12}$ and Direct Red $28,{ }^{2}$ the diffraction plane at lower angle side and that at higher angle side are perpendicular to each other. The higher angle plane of the product may thus become perpendicular to the lower angle plane.

However, there is no difference in the X-ray diffraction diagrams for DB14 and DB15 products obtained from either isotropic sample or oriented membrane sample. This means that the effects of DB14 and DB15 on the product structure are the same. For these two dyes, the only difference is methyl and methoxy groups on the skeletal structure. The interaction of the methyl groups toward the nascent MC does not differ from that of the methoxy groups to form the product. The diffraction behaviour of DB1 and DB53 product membranes is the same in all respects. ${ }^{5}$ But the orientation of DB14 and DB15 product membranes is different from that of DB1 and DB53 product membranes. ${ }^{5}$ The better orientation of the former group seems to be due to the position of sulfonate groups on the skeletal backbone, ${ }^{2,3}$ but not

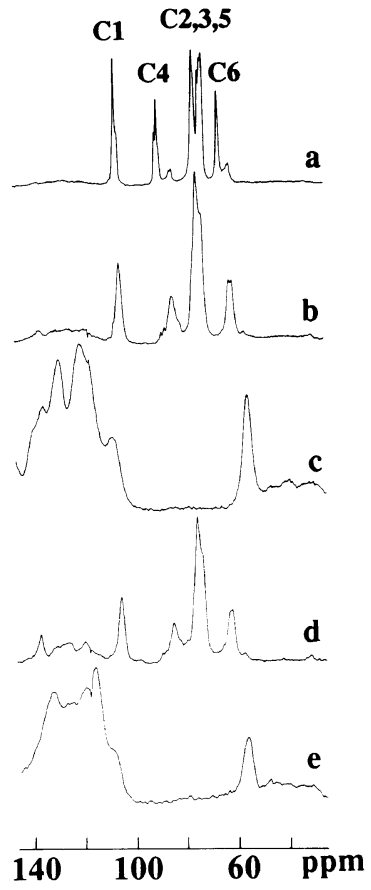

Figure 4. Solid state ${ }^{13} \mathrm{C}$ NMR spectra of wet MC, wet products and dry direct dyes. a, MC; b, DB14 product; c, DB14 powder; d, DB15 product; e, DB15 powder.

Table II. ${ }^{13} \mathrm{C}$ NMR chemical shifts (ppm) of MC and products obtained from Acetobacter-culture in the presence of DB14 or DB15

\begin{tabular}{lccccc}
\hline Sample & C1 & C4 & C2,3,5 & C6 \\
\hline MC & 106.3 & $90.2,84.7$ & $75.9,73.8,72.3$ & $66.5,62.5$ \\
DB14 & 105.2 & -85.0 & $75.2-62.2$ \\
DB15 & 105.2 & -84.9 & $75.3-61.9$ \\
\hline
\end{tabular}

methyl or methoxy groups. The dye-fibre interaction and inclusion of dye molecules between the cellulose sheets corresponding to the $(1 \overline{1} 0)$ plane are discussed in a previous paper. ${ }^{5}$

Figure 4 shows the spectra of the solid state ${ }^{13} \mathrm{C}$ NMR spectra of MC, DB14, and DB15 products and corresponding dye powders. ${ }^{13} \mathrm{C}$ chemical shifts of $\mathrm{MC}$ and the products are given in Table II.

From Figure 4, it is apparent that no resonance lines of DB14 and DB15 products overlap the lines of the corresponding dye powders except the downfield part of the $\mathrm{Cl}$ resonance line. Although the downfield part of C1 line overlaps the line of the dye powder, the ratio of dye to cellulose in the product is very low and this suggests that there is very little contribution of free dyes in the spectra of the products. The upfield $\mathrm{C} 4$ and C6 resonance lines at 84.7 and $62.5 \mathrm{ppm}$ are the noncrystalline components of $\mathrm{MC}^{4}$ The $\mathrm{C} 4$ and $\mathrm{C} 6$ resonance lines of cellulose components of products are observed only the noncrystalline component. The whole spectra of the product are broader in comparison with the spectrum of MC. The product may thus be noncrystalline and there is no ordinary hydrogen bonding between the cellulose sheets corresponding to the (110) plane of MC. The shifting of chemical shift of $\mathrm{C} 1$ resonance line upfield by $1.1 \mathrm{ppm}$ supports the absence of hydrogen bonding between the cellulose sheets in the product. ${ }^{13}$ There is 


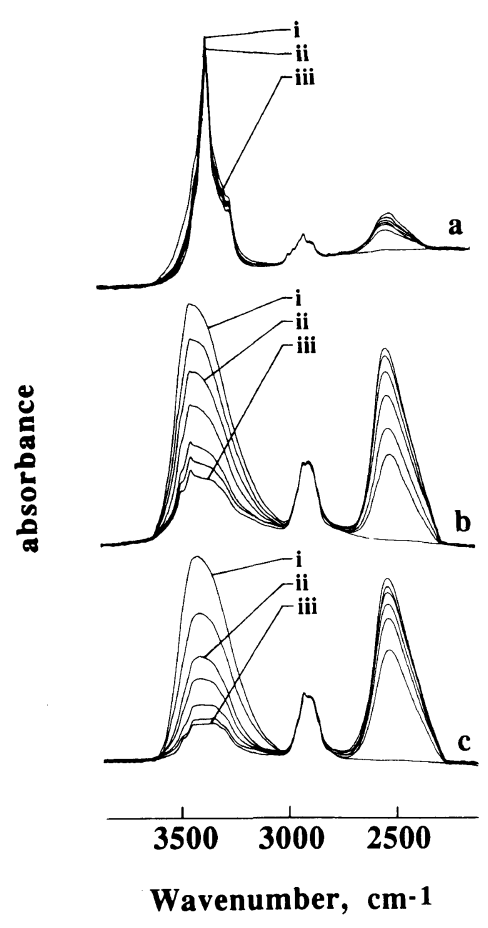

Figure 5. Change in IR spectra of the products by vapour phase deuteration at room temperature. a, MC; b, DB14 product; c, DB15 product. Deuteration time (min): i, 0 ; ii, 3; iii, 1000.

Table III. Amount of $\mathrm{OH}$ and OD groups of MC and products obtained from Acetobacter-culture in the presence of DB14 or DB15

\begin{tabular}{ccc}
\hline Sample & OH group $/ \%$ & OD group $/ \%$ \\
\hline MC & 85 & 15 \\
DB14 product & 26 & 74 \\
DB15 product & 17 & 83 \\
\hline
\end{tabular}

$\mathrm{OH}$ group, nonaccessible $\mathrm{OH}$ group; $\mathrm{OD}$ group, accessible $\mathrm{OH}$ group.

no difference between ${ }^{13} \mathrm{C}$ NMR spectra of DB14 and DB15 products as well as between those of DB1 and DB53 products. $^{5}$

The deuteration-IR spectra of DB14 and DB15 products in Figure 5 shows that most of the accessible $\mathrm{OH}$ groups transform easily to OD by vapour phase deuteration at room temperature and the absorption by OD groups appears at near $2525 \mathrm{~cm}^{-1}$. The amounts of accessible $\mathrm{OH}$ groups (OD groups) in DB14 and DB15 products are 74 and $83 \%$, respectively, which are much higher than the accessible $\mathrm{OH}$ groups of $\mathrm{MC}(15 \%)$ (Table III). This supports the noncrystallinity of the products from aspects of deuteration-IR measurements. After deuteration, the absorption by intra- and intermolecular hydrogen-bonded $\mathrm{OH}$ groups of products appeared at 3479,3439 and $3341 \mathrm{~cm}^{-1}{ }^{-9}$ which is similar to the absorption by $\mathrm{OH}$ groups of cellulose II (Figure 8). The conformational change of the chain in the cellulose component of product occurs by the removal of dyes from the products during rinsing and drying in the preparation of samples. The deuteration-IR spectra of the products are nearly identical, but different from those of DB1 and DB53 products. ${ }^{5}$ Although differences between structures of two dyes are methyl and methoxy groups, different effects on the product structure were

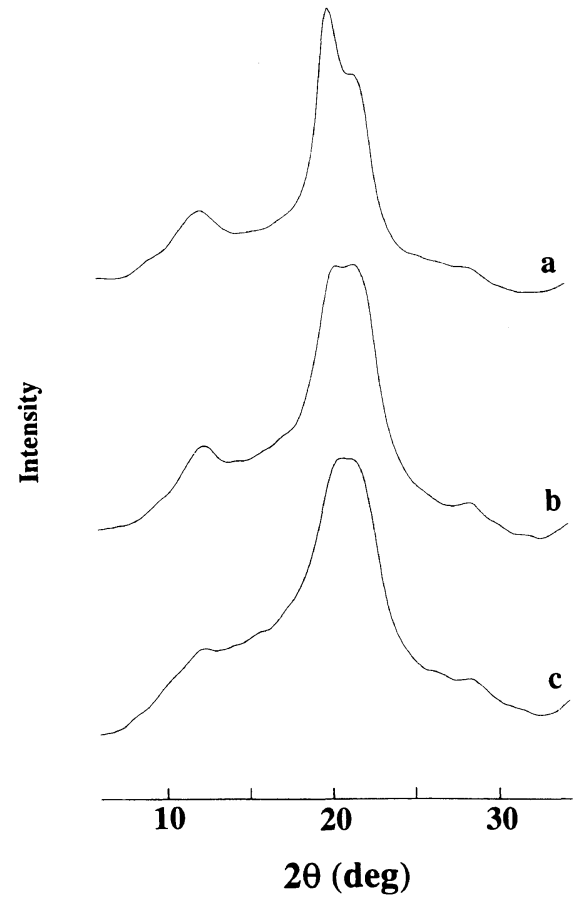

Figure 6. X-Ray diffractograms of cellulose II and celluloses regenerated from the products. a, Cellulose II; b, DB14 cellulose; c, DB15 cellulose.

not always observed. However, the spectral differences of $\mathrm{OH}$ band are observed between the groups. These differences seem to be due to the positions of sulfonate groups in the dye, but not the methyl or methoxy groups.

From X-ray measurements, the product is a crystalline complex and the dye molecule is between cellulose sheets

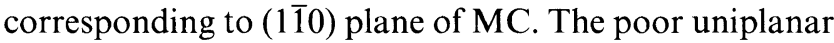
orientation of DB14 and DB15 product membranes seems to caused by the removal of some dyes from the product during rinsing. Due to removal of dyes from the product, more stable structure of cellulose II is formed through conformational change of the cellulose chains in the product. This cellulose II component in the product seems hindered to form oriented product membrane. But the product is in a noncrystalline state from the aspects of ${ }^{13} \mathrm{C}$ NMR and deuteration-IR. However, in all cases, the formation of normal hydrogen bonding between the cellulose sheets of MC is hindered by dye molecules. The crystalline character of the complex from the X-ray results suggests that there are some bonds between the dye molecule and cellulose, that form the dye-cellulose complex unit cell. ${ }^{1-3,5}$ These metastable bonds are hydrogen bonding, van der Waals forces, etc.

\section{Structure of the Cellulose Regenerated from Product Sample}

Figure 6 shows the X-ray diffraction diagrams of cellulose regenerated from DB14 and DB15 products (DB14 and DB15 cellulose) and the Table IV contains $2 \theta$ of the diffraction angles of DB14 and DB1 5 celluloses.

As apparent from Figure 6, the diffraction patterns of DB14 and DB15 celluloses are similar and similar to cellulose II. The values of the diffraction angles of these celluloses are almost the same diffraction angles of $(1 \overline{1} 0)$, (110), and (020) planes of cellulose II. Therefore, DB14 and DB15 celluloses are cellulose II. Although the 
Table IV. X-Ray diffraction angles of cellulose II and cellulose regenerated from products

\begin{tabular}{lccc}
\hline \multirow{3}{*}{ Sample } & \multicolumn{3}{c}{$2 \theta / \mathrm{deg}$} \\
\cline { 2 - 4 } & $(1 \overline{1} 0)$ & $(110)$ & $(020)$ \\
\hline Cellulose II & 12.0 & 19.8 & 21.1 \\
DB14 cellulose & 12.0 & 20.0 & 21.1 \\
DB15 cellulose & 12.3 & 20.6 & 21.1 \\
\hline
\end{tabular}

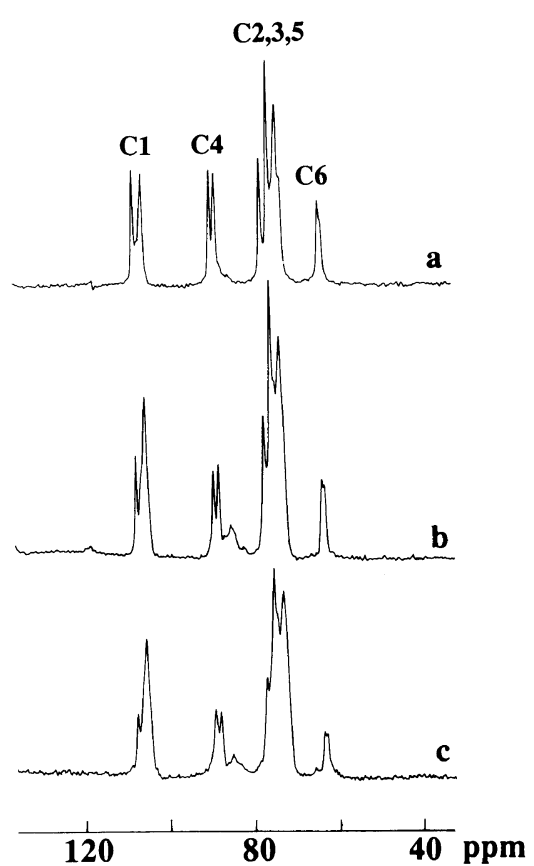

Figure 7. Solid state ${ }^{13} \mathrm{C}$ NMR spectra of crystalline components of the wet cellulose II and wet cellulose regenerated from the products. a, Cellulose II; b, DB14 cellulose; c, DB15 cellulose.

Table V. ${ }^{13} \mathrm{C}$ NMR chemical shifts of the crystalline component of cellulose II and cellulose regenerated from products

\begin{tabular}{lccccc}
\multicolumn{1}{c}{ Sample } & C1 & C4 & C2, 3, 5 & C6 \\
\hline Cellulose II & $107.5,105.3$ & $89.3,87.9$ & $77.0,75.2,72.9$ & 63.6 & 63.4 \\
DB14 cellulose & $107.9,105.6$ & $89.5,88.2$ & $77.3,75.6,73.4$ & 63.863 .2 \\
DB53 cellulose & $107.6,105.3$ & $89.2,87.9$ & $77.0,75.3,73.1$ & 63.7 & 63.3
\end{tabular}

regenerated celluloses are cellulose II, strictly speaking, there are some differences in diffraction peaks with that of cellulose II.

The solid state ${ }^{13} \mathrm{C}$ NMR spectra of the crystalline components of DB14 and DB15 celluloses shown in Figure 7 and chemical shifts listed in Table V show that the DB14 and DB 15 celluloses are cellulose II. No other differences of these celluloses from the spectrum of control cellulose II are observed, except the downfield peak of $\mathrm{C} 1$ resonance line. The weaker down field peak intensity of $\mathrm{Cl}$ resonance line of regenerated cellulose suggests that the hydrogen bonds in these celluloses are not as strong or stable as those of control cellulose II. ${ }^{13}$ The lower crystallinity of the regenerated cellulose measured by X-ray methods strongly supports this ${ }^{13} \mathrm{C}$ NMR results. The lower downfield peak intensity of $\mathrm{C} 1$ resonance line of DB15 cellulose than that of DB14

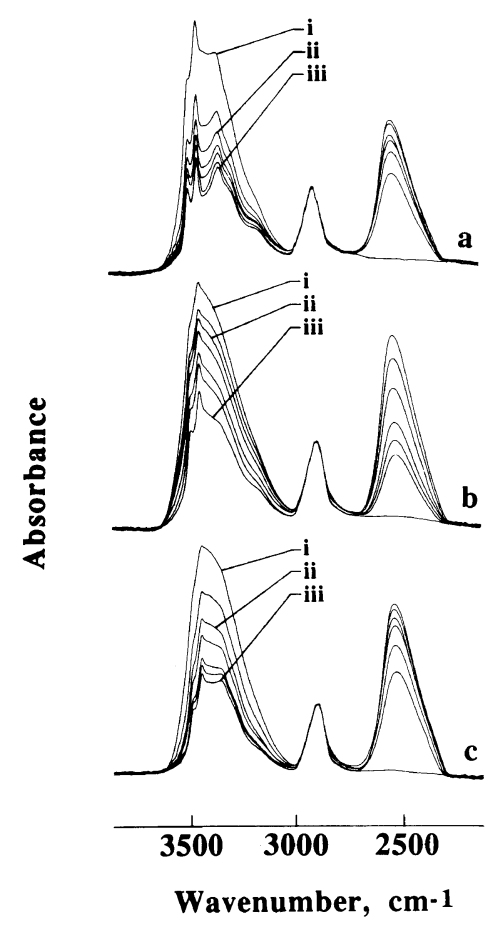

Figure 8. Change in IR spectra of cellulose II and celluloses regenerated from the products by vapour phase deuteration at room temperature. a, Cellulose II; b, DB14 cellulose; c, DB15 cellulose. Deuteration time (min): i, 0; ii, 3; iii, 1000.

Table VI. Amount of $\mathrm{OH}$ and OD groups of cellulose II and cellulose regenerated from products

\begin{tabular}{lcc}
\hline Sample & OH group $/ \%$ & OD group $/ \%$ \\
\hline Cellulose II & 45 & 55 \\
DB14 cellulose & 42 & 58 \\
DB15 cellulose & 37 & 63 \\
\hline
\end{tabular}

OH group, nonaccessible $\mathrm{OH}$ group; OD group, accessible group.

cellulose seems due to the lower crystallinity of DB15 cellulose.

The change in IR spectra of DB14 and DB15 celluloses by vapour phase deuteration at room temperature is shown in Figure 8. The amounts of accessible and nonaccessible $\mathrm{OH}$ groups are listed in Table VI.

The shapes of the absorption peaks of DB14 and DB15 celluloses by intramolecular hydrogen-bonded $\mathrm{OH}$ groups at 3484 and $3441 \mathrm{~cm}^{-1}$ and by intermolecular hydrogen-bonded $\mathrm{OH}$ groups at $3341 \mathrm{~cm}^{-1}$ are almost the same as that of cellulose II. The amounts of nonaccessible $\mathrm{OH}$ groups in DB14 and DB15 products are 26 and $17 \%$, respectively, and increase to 42 and $37 \%$, respectively. This suggests that some hydrogen bonding is newly formed in the regenerated celluloses during regeneration and appear as nonaccessible $\mathrm{OH}$ groups. The amounts of the nonaccessible $\mathrm{OH}$ groups of the regenerated celluloses show the lower crystallinity of DB14 and DB15 celluloses than that of control cellulose II $(45 \%)$. The amounts of nonaccessible $\mathrm{OH}$ groups of $\mathrm{DB}$ 15 are less than that of DB14 supports the result of ${ }^{13} \mathrm{C}$ NMR spectroscopy of DB14 and DB15.

From the results of X-ray, ${ }^{13} \mathrm{C}$ NMR and deuteration$I R$, it is apparent that there is no difference in the structures of DB14 and DB15 celluloses. This suggests that DB14 having methyl groups on the skeletal back- 
bone structure has no any different effect on the structure of the regenerated cellulose in comparison with DB15 having methoxy groups on the same skeletal structure. The effects of these dyes on the nascent structure of MC are almost the same those of DB1 and DB53. ${ }^{3}$

\section{CONCLUSION}

The product from Acetobacter-culture in the presence of DB14 or DB15 is a crystalline dye-cellulose complex and the dye molecule is between the cellulose sheets corresponding to the (110) plane of MC. The cellulose regenerated from the product is cellulose II. Although DB14 contains two methyl groups instead of two methoxy groups on the same biphenylenebis(azo) skeletal backbone in DB15, no other different effects of these different groups in dye on the structures of the product and their regenerated celluloses were found.

\section{REFERENCES}

1. A. Kai and Md. I. H. Mondal, in "Biomedical Functions and Biotechnology of Natural and Artificial Polymers," M. Yalpani, Ed., ATL Press, Inc., Science Publishers, Shrewsbury, MA, 1996, p 77.

2. A. Kai and Md. I. H. Mondal, Int. J. Biol. Macromol., 20, 221 (1997).

3. Md. I. H. Mondal and A. Kai, Int. J. Biol. Macromol., in press.

4. A. Kai, P. Xu, F. Horii and S. Hu, Polymer, 35, 75 (1994).

5. Md. I. H. Mondal and A. Kai, Polym. J., 30, 78 (1998).

6. A. Kai, H. Kido, and N. Ishida, Chem. Lett., 949 (1990).

7. S. Hestrin and M. Schramm, Biochem. J., 58, 345 (1954).

8. D. A. Torchia, J. Magn. Reson., 30, 613 (1978).

9. S. Okajima and A. Kai, J. Polym. Sci., A-1, 6, 2801 (1968).

10. A. Kai and P. Xu, in "Cellulosics: Chemical, Biochemical and Material Aspects," J. F. Kennedy, G. O. Phillips, and P. A. Williams, Ed., Ellis Horwood Ltd., London, 1993, p 2325.

11. M. Takai, Y. Tsuta, J. Hayashi, and S. Watanabe, Polym. J., 7, 157 (1975).

12. A. Kai and P. Xu, Kobunshi Ronbunshu, 48, 449 (1991).

13. F. Horii, A. Hirai, and R. Kitamaru, Polym. Bull., 8, 163 (1982). 